\title{
The best spot to park a CAR
}

Cancer immunotherapy using $\mathrm{T}$ cells engineered to express chimeric antigen receptors (CARs) has achieved exciting results in patients with B-cell malignancies. In a recent report in Nature, Eyquem et al. ${ }^{1}$ sought to improve the technology. They inserted the CAR sequence into $\mathrm{T}$ cells at the TRAC locus, encoding the $\mathrm{T}$-cell receptor (TCR) $\alpha$ chain constant region, under the control of the gene's endogenous promoter, rather than rely on semi-random retroviral integration and exogenous promoters. The resulting $\mathrm{T}$ cells had higher anti-tumor efficacy in mice, persisted longer in vivo and revealed mechanistic advantages of precisely regulated CAR expression.

CARs are synthetic proteins that combine a tumor-antigen-binding extracellular domain and a T-cell-activating intracellular domain. In current protocols to manufacture CAR-T cells, a construct encoding a CAR is delivered to a patient's T lymphocytes ex vivo using a retroviral vector. Semirandom viral integration into the genome leads to a heterogeneous population of cells that differ in CAR expression levels and sites of insertional mutagenesis. A better- defined cellular product is expected to offer improved safety and efficacy.

Eyquem et al. ${ }^{1}$ used CRISPR technology to insert a B-cell-specific CAR into the TRAC locus, putting the CAR under the control of the endogenous TRAC promoter and simultaneously disrupting the endogenous TCR by knocking out one of its multiple subunits. This elegant move addressed two possible shortcomings of CAR-T cells-variable CAR expression levels and interference from TCR activity. Compared with retrovirally transduced CAR-T cells, the gene-edited T cells showed more uniform CAR protein expression and enhanced tumor rejection in a mouse model of acute lymphoblastic leukemia.

Earlier research had suggested that the low efficacy of CAR-T cells may stem from excessive tonic signaling (low-affinity interactions of the TCR with self-peptides) or CAR-T-cell exhaustion. Consistent with those findings, Eyquem et al. ${ }^{1}$ showed that gene-edited $\mathrm{T}$ cells had an improved signaling profile compared with conventional CAR-T cells: without antigen exposure, their phenotype was more naive and, upon repeated stimulation, they differentiated later. "The fact that

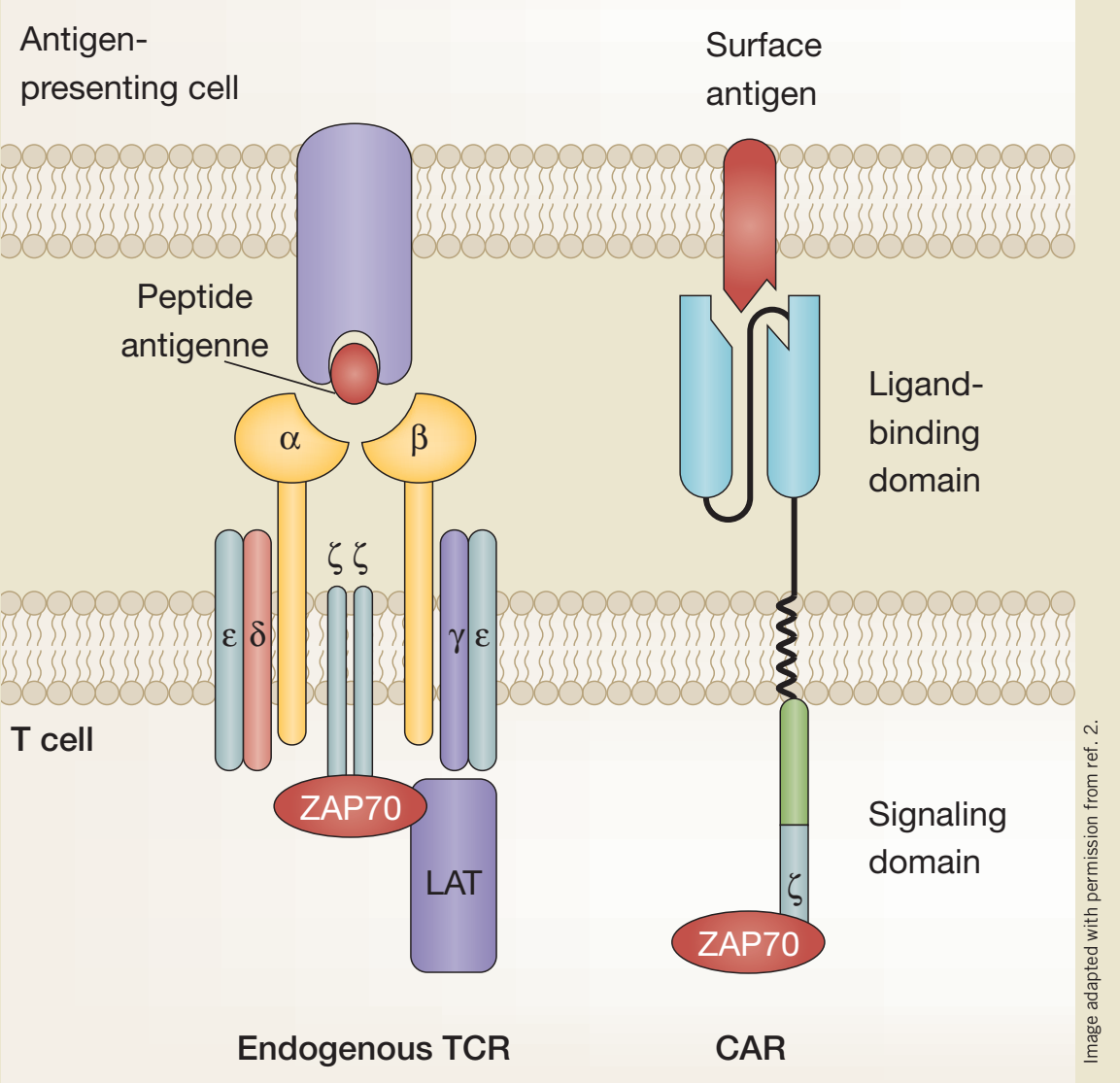

the gene-edited cells remain active for a long time and that they don't seem to have tonic signaling may be important for long-term control of the disease," says Helen Heslop of Baylor College of Medicine in Houston, Texas.

In an effort to distinguish the effects of CAR expression regulation from those of endogenous TCR disruption, the team compared cells carrying the CAR at different loci and under the control of different promoters. Notably, when exposed to antigen, the TRAClocus-edited cells had a unique pattern of CAR internalization, degradation and delayed reexpression, with CAR levels never exceeding the initial, moderate baseline.

Taken together, the results of Eyquem et al. ${ }^{1}$ suggest that optimal antitumor performance may require both TCR disruption (perhaps to curb tonic signaling) and fine-tuning of CAR expression-and that both can be achieved by inserting the CAR at the TRAC locus. "This study exemplifies how CAR-T cells can be a very precise tool to learn biology," says Crystal Mackall of Stanford University in Stanford, California.

Whether the observations of Eyquem et al. ${ }^{1}$ are broadly applicable to other types of CARs and malignancies remains to be determined. It will be important to test the approach against solid tumors, which have proved difficult to target with CAR-T cells. Gene-edited CAR-T cells may also be safer than conventional cells because they are less likely to undergo oncogenic transformation or to cause unintended TCR-mediated immune reactions.

\section{Katarzyna Marcinkiewicz,} Locum Assistant Edito

1. Eyquem et al. Nature 543, 113-117 (2017).

2. Fesnak, A.D., June, C.H. \& Levine, B.L. Nat. Rev. Cancer 16, 566-581 (2016). 